\title{
Oscillations and exact states of neutrinos in a magnetic field
}

\author{
Artem Popov $^{* a}$, Alexander Studenikin ${ }^{a b}$ \\ ${ }^{a}$ Department of Theoretical Physics, Moscow State University \\ 119991 Moscow, Russia \\ b Joint Institute for Nuclear Research \\ Dubna 141980, Moscow Region, Russia \\ E-mail: ar.popovephysics.msu.ru, studenikesrd.sinp.msu.ru
}

\begin{abstract}
Within the recently proposed new approach $[1,2]$ we investigate the problem of neutrino oscillations in a magnetic field. Probabilities of relativistic neutrino flavour $v_{e}^{L} \leftrightarrow v_{\mu}^{L}$, spin $v_{e}^{L} \leftrightarrow v_{e}^{R}$ and spin-flavour $v_{e}^{L} \leftrightarrow v_{\mu}^{R}$ oscillations are calculated on the base of the exact neutrino stationary states in the magnetic field accounting for the whole set of possible conversions between four neutrino states. It is shown that there is an entanglement between neutrino flavour and spin oscillations and in the general case it is not possible to consider these two types of neutrino oscillations separately. In particular, the probability of flavour oscillations exhibits quite complicated dependence on vacuum and magnetic frequencies.
\end{abstract}

XXXIX International Conference on High Energy Physics

4-11 July, 2018

Seoul

${ }^{*}$ Speaker. 


\section{Neutrino interaction with an arbitrary magnetic field}

We start with the consideration of flavour neutrinos with two chiralities accounting for mixing in the case of two flavours (generalization on the three-flavour case is trivial) $v_{e}^{L(R)}=v_{1}^{L(R)} \cos \theta+$ $v_{2}^{L(R)} \sin \theta, \quad v_{\mu}^{L(R)}=-v_{1}^{L(R)} \sin \theta+v_{2}^{L(R)} \cos \theta$, where $v_{i}^{L(R)}$ are the chiral neutrino mass states, $i=1,2$. For the relativistic neutrinos the chiral states approximately coincide with the helicity states $v_{i}^{L(R)} \approx v_{i}^{h^{-}\left(h^{+}\right)}$. Note that the helicity mass states $v_{i}^{h^{-}\left(h^{+}\right)}$are not stationary states in the presence of a magnetic field. With a view to describe neutrino oscillations within our method, we shall expand $v_{i}^{h^{-}\left(h^{+}\right)}$over the neutrino stationary states $v_{i}^{-(+)}$in the presence of a magnetic field.

The wave function $v_{i}^{s}(s= \pm 1)$ of a massive neutrino stationary states in the presence of a magnetic field can be found as the solution of the Dirac equation

$$
\left(\gamma_{\mu} p^{\mu}-m_{i}-\mu_{i} \mathbf{\Sigma} \mathbf{B}\right) v_{i}^{s}(p)=0
$$

where $\mu_{i}$ are magnetic moments of neutrinos. In the discussed two-neutrino case the possibility for a nonzero neutrino transition moment $\mu_{i j}(i \neq j)$ is not considered and two equations for two neutrinos states $v_{i}^{s}$ are decoupled. In order to classify neutrino stationary states, we use the spin operator

$$
\hat{S}_{i}=\frac{m_{i}}{\sqrt{m_{i}^{2} B^{2}+p^{2} B_{\perp}^{2}}}\left[\boldsymbol{\Sigma} \boldsymbol{B}-\frac{i}{m_{i}} \gamma_{0} \gamma_{5}[\boldsymbol{\Sigma} \times \boldsymbol{p}] \boldsymbol{B}\right],
$$

which is an integral of motion. By decomposing a massive state wavefunction over the stationary states, we can formulate the problem of neutrino oscillations in a magnetic field in quite a similar to the vacuum case form. In particular, we've introduced a generalization of the mixing matrix on the case of magnetic field.

The probability of flavour neutrino oscillations in a magnetic field in the two-flavour case is

$$
P_{v_{e}^{L} \rightarrow v_{\mu}^{L}}(t)=\sin ^{2} 2 \theta\left\{\cos \left(\mu_{1} B_{\perp} t\right) \cos \left(\mu_{2} B_{\perp} t\right) \sin ^{2} \frac{\Delta m^{2}}{4 p} t+\sin ^{2}\left(\mu_{+} B_{\perp} t\right) \sin ^{2}\left(\mu_{-} B_{\perp} t\right)\right\},
$$

where $\mu_{ \pm}=\frac{1}{2}\left(\mu_{1} \pm \mu_{2}\right)$. In particular, in the case $\mu_{1}=\mu_{2}$ eq.(3) can be expressed as $P_{v_{e}^{L} \rightarrow v_{\mu}^{L}}^{c u s t}(1-$ $P_{v_{e}^{L} \rightarrow v_{e}^{R}}^{\text {cust }}$, where $P_{v_{e}^{L} \rightarrow v_{\mu}^{L}}^{\text {cust }}$ and $P_{v_{e}^{L} \rightarrow v_{e}^{R}}^{\text {cust }}$ are the probabilities of flavour and spin oscillations obtained in the customary approach. Similar result have been obtained in [3]. Our conclusion is that due to an entanglement it is not possible to consider spin and flavour oscillations separately.

This work is supported by the Russian Basic Research Foundation grants No. 16-02-01023 and 17-52-53-133.

nnn

\section{References}

[1] A. Popov and A. Studenikin, Neutrino oscillations and exact eigenstates in magnetic field, arXiv:1803.05755 [hep-ph].

[2] A. Dmitriev, R. Fabbricatore and A. Studenikin, Neutrino electromagnetic properties: new approach to oscillations in magnetic fields, PoS CORFU 2014 (2015) 050, arXiv:1506.05311 [hep-ph].

[3] P. Kurashvili, K. Kouzakov, L. Chotorlishvili and A. Studenikin, Spin-flavor oscillations of ultrahigh-energy cosmic neutrinos in interstellar space: The role of neutrino magnetic moments, Phys. Rev. D 96 (2017) 103017, arXiv:1711.04303 [hep-ph]. 